\title{
ACTP: An Advanced Data Collection Protocol based on CTP
}

\author{
Xingming Sun ${ }^{1,2,3}$, Lei Gao ${ }^{1}$, Baowei Wang ${ }^{1,2,3}$ and Jian Zhang ${ }^{1,2}$ \\ ${ }^{1}$ School of Computer and Software, Nanjing University of Information Science \& \\ Technology, Nanjing 210044, China \\ ${ }^{2}$ Jiangsu Engineering Center of Network Monitoring, Nanjing 210044, China \\ ${ }^{3}$ Jiangsu Collaborative Innovation Center on Atmospheric Environment and \\ Equipment Technology,Nanjing 210044, China \\ sunnudt@163.com,leifgao@163.com,wbwfirst@163.com, \\ Jianzhang_neu@163.com
}

\begin{abstract}
In wireless sensor networks, the main work of nodes is for data collection and transmission. CTP (Collected Tree Protocol) had been widely applied, and proved to be reliable, robust, efficient data collection protocol. Its independent with hardware and the data delivery rate is relatively high, but CTP also has some shortcomings: the protocol only relied on the ETX value of next hop node selection, there need other factors to consider. In the original CTP protocol, the energy consumption of a node with a better ETX value was too fast. It would cause the whole network time decreased quickly. Then, the node with better ETX value may have low package rate, and would affect the delivery rate of the whole networks. This paper was aimed at this two aspects of CTP, based on the original CTP, the probability of selection mechanism was introduced, proposed ACTP, lead the node load equilibrium and improved the delivery rate. Through the experimental data of TelosB nodes in real analysis, we had improved the whole network time and increased the number of successful packets delivery.
\end{abstract}

\section{Introduction}

Data collection is the basic work of wireless sensor networks [1-4]. Data collection protocol will directly affect the data collection performance of various practical applications. Routing protocols can be divided into two types: flat routing and cluster routing. CTP belongs to the flat routing; its basic idea is to establish a sink node as its collection tree root. The root node is sink node (base station node). Other nodes will forward their collected data to the node that closer to the sink node. We call this node as "next hop node". The next node will repeat this action to transfer data until the collected data successfully passed to the sink node. Compared with other routing protocols, CTP [5-9] has shown better performance in practical applications of WSN (Wireless Sensor Networks).

However, some problems also existed in CTP. When it works, CTP strategy selects the optimal path every time. It will accelerate the energy consumption on this path. If things go on like this, it will shorten the life of the whole networks [10]. As we all known, nodes generally adopt a dry battery and deployed in the field, artificial battery replacement is very troublesome. In order to extend the entire wireless sensor networks lifetime, we consider import some other factors on the basis of the CTP. The factors are as follows: the rate of transfer packets. The rate of transfer packets is the ratio of the sends out packets and all received packets. The blocking situation of the whole network will be improved if we take this factor into consideration.

In order to solve the above problem, we proposed a new routing protocol named "ACTP (Advanced Collection Tree Protocol)". ACTP studies on how to extend the WSN 
networks lifetime and how to increase the number of successfully transfer packets. Based on the link quality estimator of CTP, we take the remaining energy and the rate of transfer packets into consideration to achieve our goal.

In the second chapter, we mainly reviewed the working procedure of CTP. The third chapter generally introduced the working procedure of ACTP. In the fourth chapter, we made some tests through TelosB [11] nodes. Through analyzing the rate of transfer packets and the networks lifetime, we approved that ACTP is much better than the original CTP. Finally, we summarized our arguments and some future work in fifth chapter.

\section{Related Work}

ACTP is an improvement of CTP. CTP named Collection Tree Protocol. The nodes transfer their collected data one by one to the sink node through this tree. Each node maintains the bidirectional link quality evaluation value between itself and its neighbor node. We called this value as ETX value [12]. They choose their parent nodes according to the ETX value. The least ETX value, the best link quality has. After chosen their parent nodes, they transferred their data to sink node one by one through each node's parent node.

CTP Protocol can be divided into three parts, including the link quality estimator, the routing engine and the forwarding engine. The link estimator is responsible for establishing and maintaining the current node and its neighbor node's link quality information. It maintains a neighbor list and provides the information for routing establishment. It implements its function though LEEP (Link Estimation Exchange Protocol). The routing engine is responsible for initiating the establishment of routing and choosing the best link quality routing to the sink node. It also maintenances the routing table and timely updates the routing information. Forwarding engine implements data transfer and the maintenance of transfer queue of local packets and transmits packets. They select the appropriate time to send out the head packets located in queue head to their parent node [13].

\subsection{Link Quality Estimator}

The link quality estimator estimates its bidirectional link quality with its neighbor nodes by calculating the successful rate of broadcasting the LEEP frame (beacon frame). Link quality estimator counts the packets that forwarded to the nodes and also counts the packets that send out. EETX (Extra Expected Transmissions) Value is the ratio of the input packets and output packets. We evaluate the link quality by ETX value.

\subsection{Routing Engine}

CTP uses the ETX value as its routing gradient. The sink node's ETX value is 0 . The other nodes' EXT value are their parent nodes' ETX value adds the ETX value that their parent nodes to sink node. Nodes broadcast their routing statement information periodically and update the ETX value that their neighbor nodes to sink node. When the routing table had been updated completely, nodes choose their next hop node according to who has the minimum EXT value. As the routing gradient, ETX ensures the data transmission along the path of minimum value. It facilitates the detection of routing loops.

\subsection{Forwarding Engine}

Forwarding engine maintains a packet send queue that mixed locally generated packets with forwarded packets. It determines when to transmit the data packets to 
the next hop node and whether to retransmit. At the same time, it is responsible for detecting the routing loops and single hop repeated transmissions.

\subsection{Some Research Results of WSN}

BCTP [14] wanted to prolong the wireless sensor networks lifetime through three steps to solve the problem. First, hot spot detection when detected hot spot then load migration and last integration with original CTP.

In E-CTP [15], the authors proposed that E-CTP would detect the residual voltage before the original ETX selection. When the voltage is greater than a certain threshold, we adopted the original selection strategy. But when the voltage is less than the threshold value, E-CTP calculates a coefficient greater than 1.The original ETX value multiplied by the coefficient. So the ETX value will change by the residual voltage and eventually changed the next hop node selection strategy.

There were also some other research: such as underwater sensor networks [16], they proposed a novel routing protocol for underwater sensor networks. Tree networks [17], they wanted to construct a tree network for homogeneous wireless sensor networks.

\section{Design of ACTP}

In order to solve these problems, we import the probability selection mechanism into the original CTP protocol. By adding the residual voltage of each node and its rate of packets out, the lifetime of WSN networks will be prolonged and the success number of packets that transmitted back to the sink node will be increased.

The basic idea of ACTP is to establish a tree topology with the sink node as the root. The other nodes choose a parent from the neighbors as the next hop node. On the one hand the node collects data itself, on the other hand the node forward s data to other nodes, and through the tree to the base station. Each node maintains the bidirectional link quality evaluation value between itself and its neighbor node, and its routing table records its residual voltage, transfer packets of neighbor nodes. We choose the next hope node according to the bidirectional link quality evaluation and choose some neighbor nodes in a certain range as the candidate nodes. In this group of candidate nodes, we calculate the comprehensive probability according to the probability of residual voltage and the rate of transfer packets to. Finally, we select the next hop node by calculating the comprehensive probability.

\subsection{The Detailed Steps}

First, select the nodes that ETX value within a certain range as the candidate nodes. There's not much difference in link quality between the nodes closer in ETX value. The original CTP strategy will only choose the node which has the minimum ETX value as its next hop node. The smaller the ETX value the better the link quality between the two nodes.

We calculate the ETX value between the ranges of $\left[E T X_{\min }, \alpha E T X_{\min }\right]$. ETX $X_{\min }$ is the minimum value of the neighbor nodes and also is the optimal value. Where alpha is a real number greater than 1 . After many experiments, 1.3 is the best value of alpha.

Second, after the computation of first step, if the candidate node has only one node, we are in accordance with the original CTP strategy to select the next hop node. But if there is more than one candidate node, we compute the node's rate of residual voltage and the rate of transfer packets. We combined the two probabilities together to get each node's probability. The formula is $\mathrm{P}=0.5 P_{\text {voltage }}+0.5 P_{\text {packet rate }} . P_{\text {voltage }}$ is the name of the probability of residual 
voltage. $P_{\text {packet rate }}$ is the probability of the rate of transfer packets. We select the next hop node among the candidate nodes according the value of $\mathrm{P}$.

\subsection{The Computation of the Probability of Residual Voltage}

We will get the candidate node voltage value respectively when there's more than one candidate node. The higher is the candidate node's value, the more we tend to choose the node as the next hop node. We know, the probability of the residual voltage is proportional to each node's residual voltage. The formula of the residual voltage probability is $\frac{V_{i}}{\sum_{k \in F T_{j}} V_{k}} . \mathrm{V}_{\mathrm{i}}$ is the current candidate node's voltage. The denominator of the formula represents the sum of all the candidate nodes' voltage in the routing table. By this formula, we calculated the ratio of each candidate nodes residual voltage in the sum of all the candidate nodes residual voltage. The probability is bigger the probability of being chosen is big.

\subsection{The Calculation of the Rate of Transfer Packets}

First of all, we should explain what is the rate of transfer packets? WSN nodes need to transfer the collected data packets to the other nodes. Therefore, there is an inbound queue and an outbound queue of each node. We can calculate the total number of packets into the inbound queue and how many packets had send out from the outbound queue. The formula of the rate of transfer packets is $\frac{\mathrm{PKG}_{\text {out }}}{\mathrm{PKG}_{\text {in }}} . \mathrm{PKG}_{\text {out }}$, $\mathrm{PKG}_{\mathrm{in}}$ respectively represents the node's packet number of enter in and out. By this formula we can see that the bigger the rate of transfer packets the more the incoming packets had been transmitted. The network congestion is also related to the ratio. Small ratio represents the serious network congestion. It represents large number of incoming packets are still in the queue. As the same with residual voltage above, we choose the next hop node according to the better rate of transfer packets. The probability of packets rate is proportional to the rate of transfer packets. The formula of the rate of transfer packets is $\frac{\mathrm{TPR}_{\mathrm{i}}}{\sum_{\mathrm{k} \in \mathrm{FT}_{\mathrm{j}} \mathrm{TPR}_{\mathrm{k}}}} . \mathrm{TPR}_{\mathrm{i}}$ represents the send out packets rate of the candidate node. The denominator formula represents the sum of all the candidate nodes' send out packets rate in the routing table. By this formula, we calculated the probability of sends out packets rate for each candidate node.

\subsection{The Fusion of these Two Probabilities}

We calculated the probability of the residual voltage and the rate of transfer packets, then we got a combined formula: $\mathrm{P}=0.5 P_{\text {voltage }}+0.5 P_{\text {packet rate }}$. These two effectors both important so we set the coefficient of two in front of the probability is 0.5 . After gotten the comprehensive probability we selected the next hope node according the sum probability.

The whole decision process as shown in Figure1: 


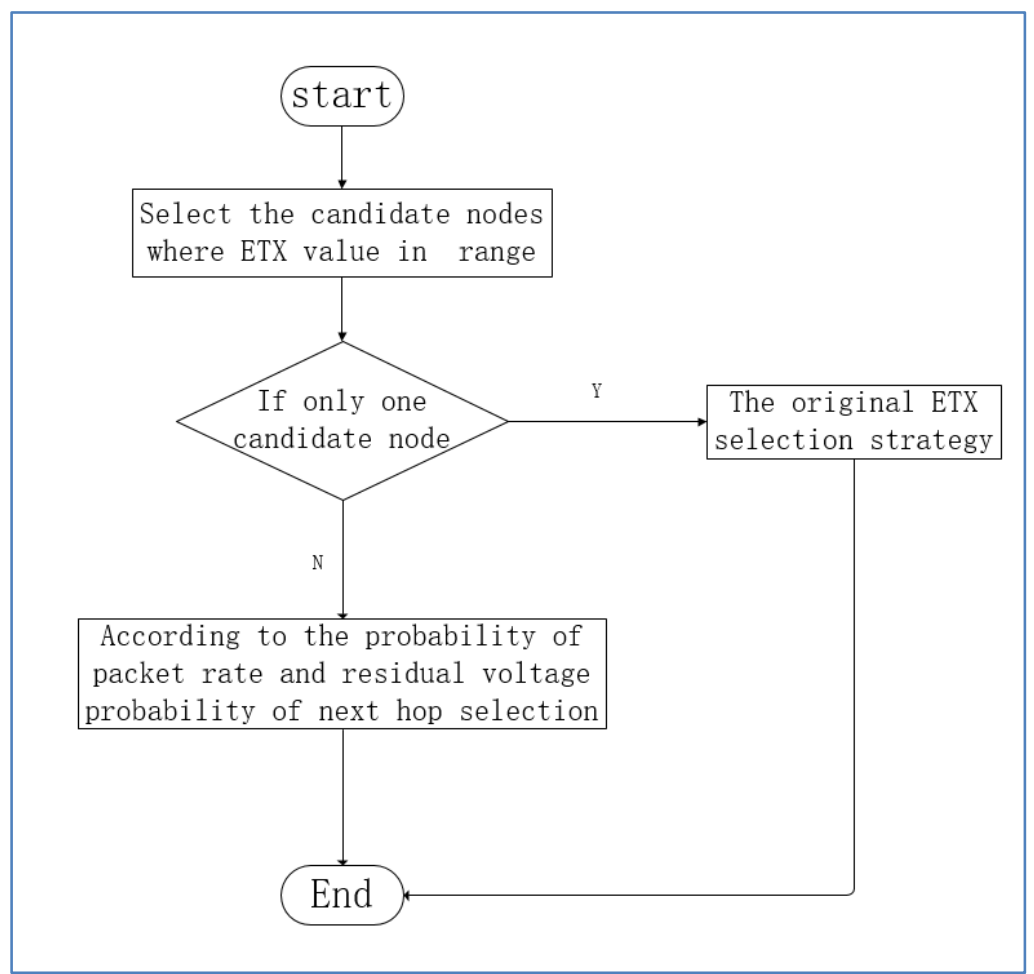

Figure 1. Decision Process

\section{Results and Evaluation}

\subsection{Testbeds}

In order to verify the effect of our ACTP, we wrote the ACTP into TelosB nodes. There're one node connected with the computer as the sink node. Sink node was responsible for receive all the transferred data and transmitted to computer. TinyOS [18] is designed by the University of California at Berkeley. It based on an embedded open source platform. TelosB node is a cost-effective mainstream node because of its low power consumption, convenient to apply in many applications and strong robust in hardware and software.

We had invested ten nodes number one to number ten as our experiment object. The node numbered 0 is our sink node. The layout of these ten nodes is shown at Figure 2 and Figure 3:

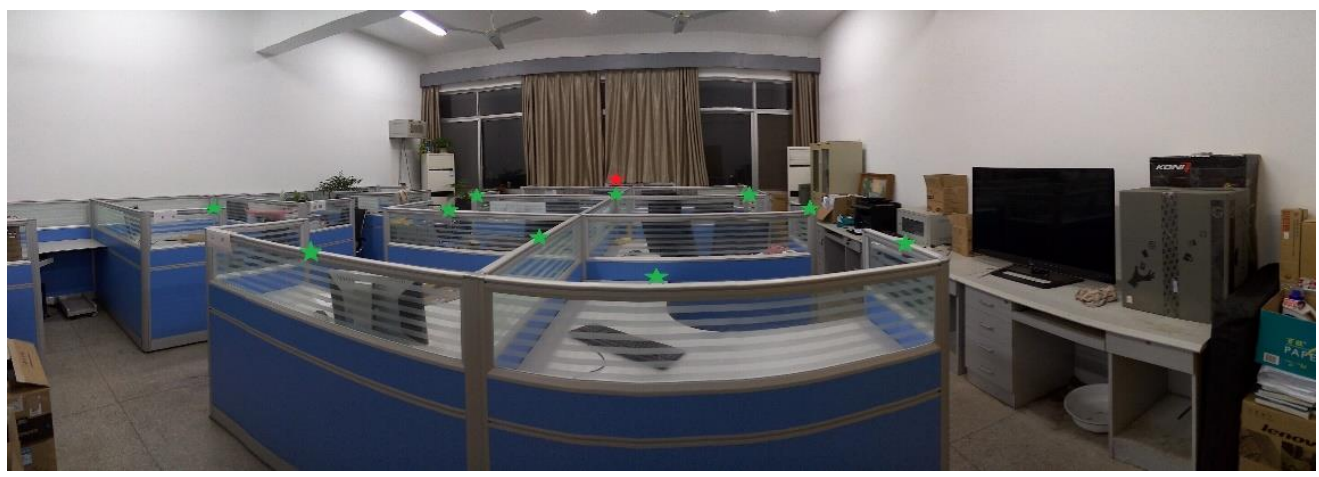

Figure 2. The Environment of Node's Deployment 


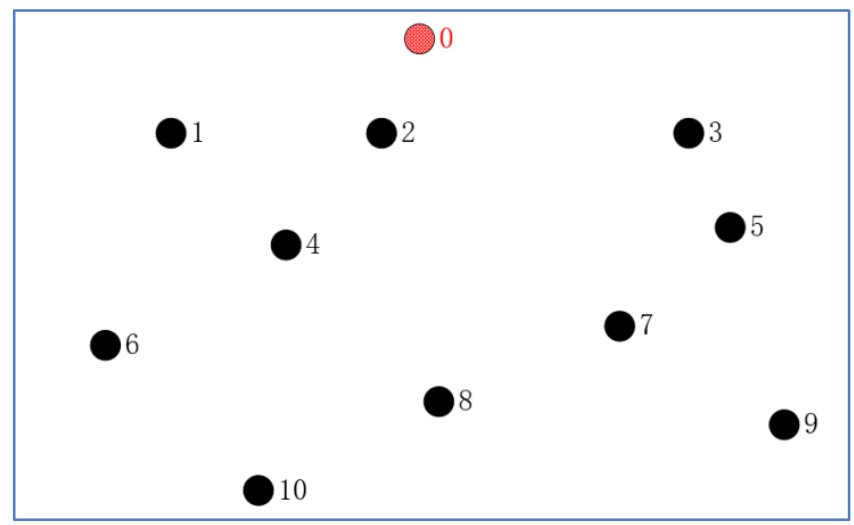

Figure 3. The Topology of the Nodes

\subsection{Results and Evaluation}

Through the analysis of our data, we found that when the node voltage below $2.3 \mathrm{~V}$, the collected data would appear larger error. So we toke $2.3 \mathrm{~V}$ as TelosB node's death voltage.

First, look at the whole working time of the nodes in Table1.Figure4 is the comparison chart of each node.

\section{Table 1. The Whole Working Time of the Nodes}

\begin{tabular}{|c|r|r|r|r|r|r|r|r|r|r|r|}
\hline The protocol name Node number & 1 & 2 & 3 & 4 & 5 & 6 & 7 & 8 & 9 & 10 & Average \\
\hline CTP & 6800 & 6905 & 6205 & 6490 & 6950 & 7005 & 6470 & 6870 & 6880 & 7110 & 6768 \\
\hline ACTP & 6950 & 7005 & 6850 & 7000 & 6840 & 6940 & 7100 & 6895 & 7150 & 7330 & 7006 \\
\hline
\end{tabular}

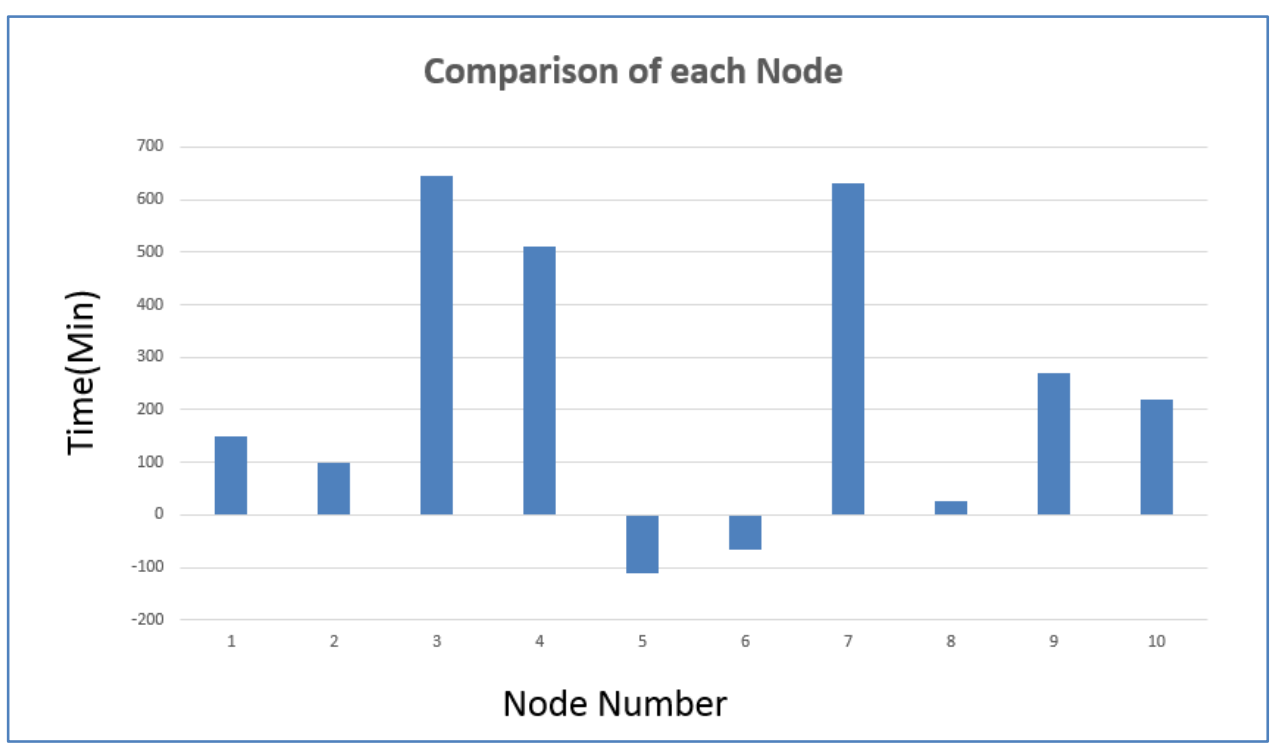

Figure 4. Comparison of each Node

From Table 1, we seen that the ten nodes who running in the ACTP protocol had working 238 minutes longer than the 10 nodes running in original CTP. ACTP protocol was prolonged the nodes lifetime and it was better than CTP. In order to compare the working lifetime of each node, our analysis is presented in Figure 5. Each histogram shows the time difference in ACTP and CTP. From Figure 4, we see 
that there are seven nodes extend their working lifetime and only three nodes shorter in ACTP. Among them, node three, node four, and node seven's working lifetime is relatively extensive. Through the analysis of our topology, node three, node four and node seven bear task too much as the next hop node in original CTP. Then leading the voltage consumption lost too fast. However, we considered more factors in ACTP. Other node shares the same probability of node three four and seven, ultimately this will prolong the lifetime the WSN networks.

Figure 5 and Figure6 give respectively the comparison of node four and node seven voltages in ACTP and CTP.

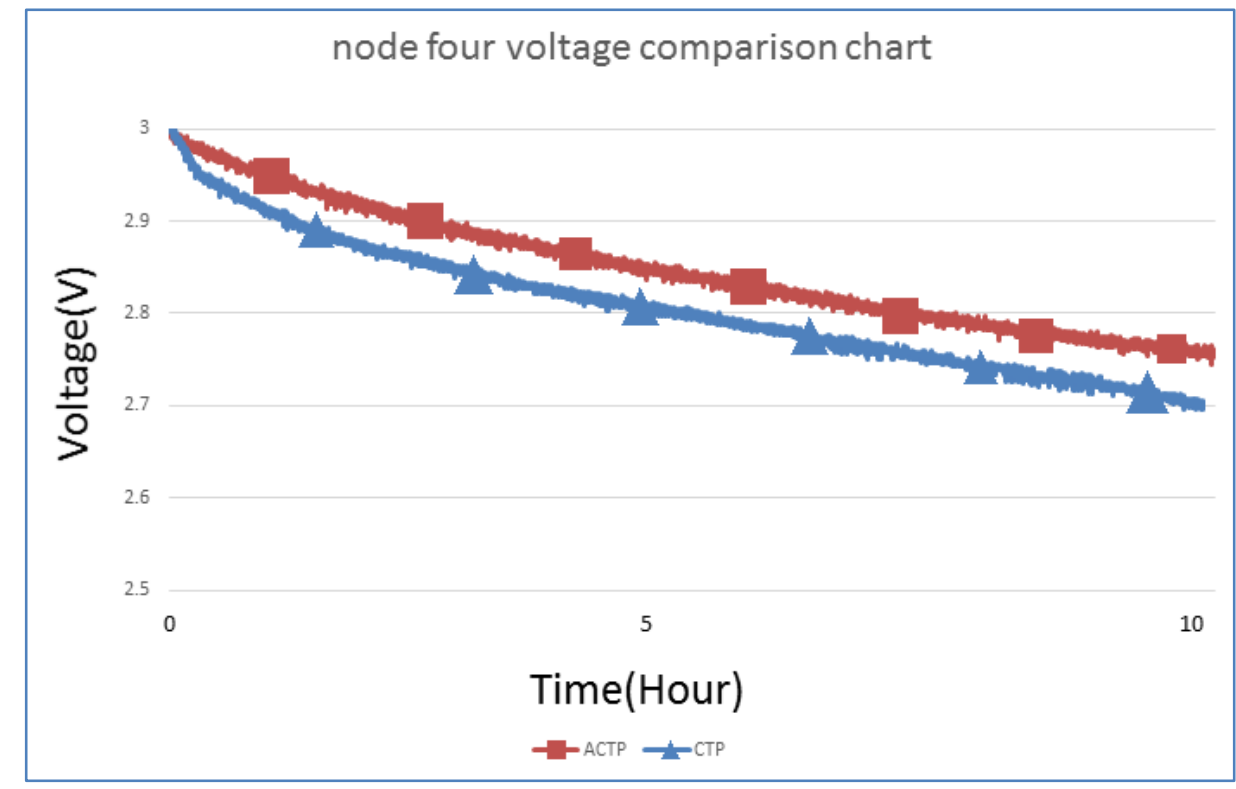

Figure 5. Node Four Voltage Comparison Chart

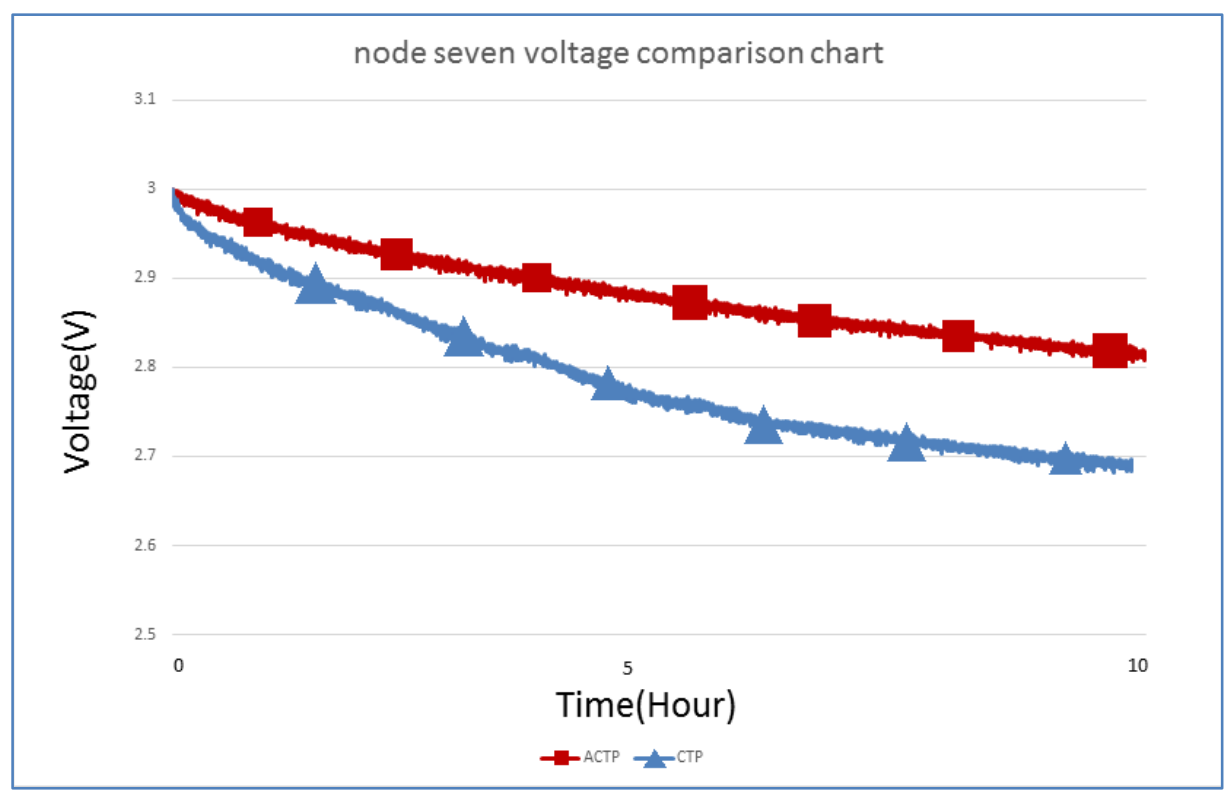

Figure 6. Node Seven Voltage Comparison Chart

Figure 7 is the long ling chart of these two nodes in two protocols. 


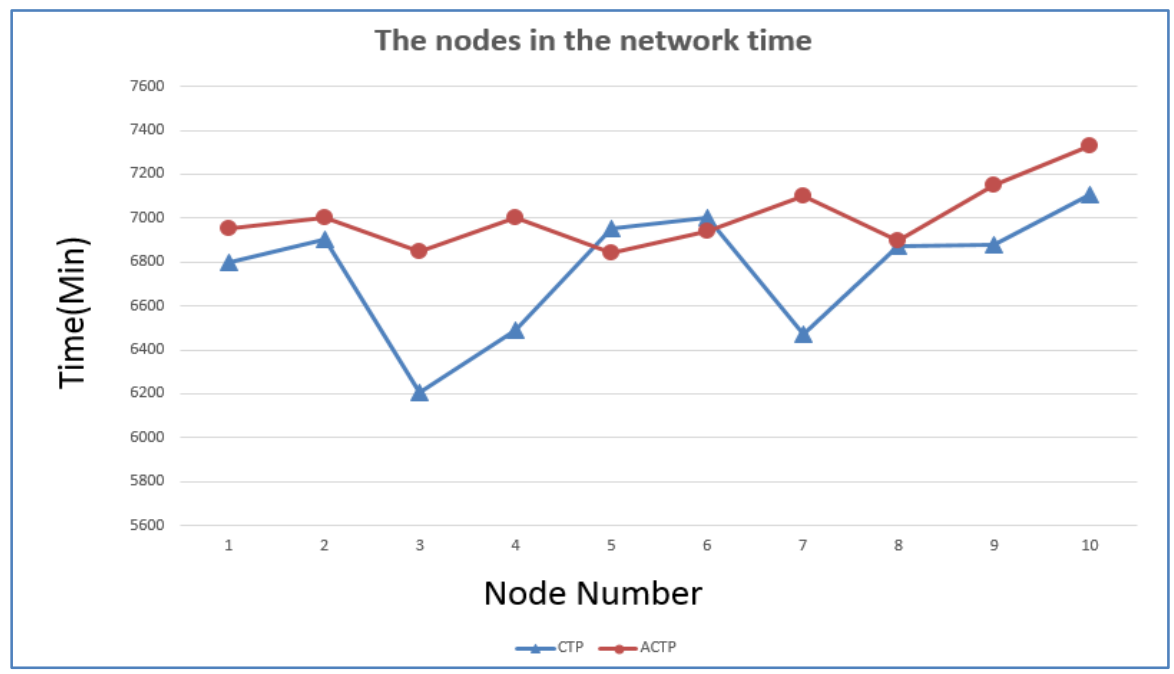

Figure 7. Nodes in the Network Time

From the Figure 8, compared with CTP, the chart of ACTP is smoother. Described in ACTP, the lifetime of each node is close with each other. But in CTP, the life time of each node is high or low. We respectively calculate the standard deviation to verify the lifetime. The network length of standard deviation is 143.628 in ACTP. But in CTP, the number is 270.565 . Through this data shown, we know that the time of death in ACTP is more concentrated. ACTP is more loader balanced.

Table 2. The Number of Received Date Packets that each Node received in Ten Hours

\begin{tabular}{|l|r|r|r|r|r|r|r|r|r|r|}
\hline & 1 & 2 & 3 & 4 & 5 & 6 & 7 & 8 & 9 & 10 \\
\hline CTP & 35888 & 35013 & 35112 & 35915 & 35698 & 36433 & 35642 & 36011 & 35089 & 34976 \\
\hline ACTP & 37303 & 36641 & 36524 & 36446 & 36124 & 37663 & 36987 & 37524 & 37909 & 37215 \\
\hline Differe & 1415 & 1628 & 1412 & 531 & 426 & 1230 & 1345 & 1513 & 2820 & 2239 \\
\hline
\end{tabular}

Table2 recorded the number of received data packets that node received in ten hours. In this form we can see that the successful transfer packets in ACTP are much more than in CTP. Figure8 shows the comparison of data packet number.

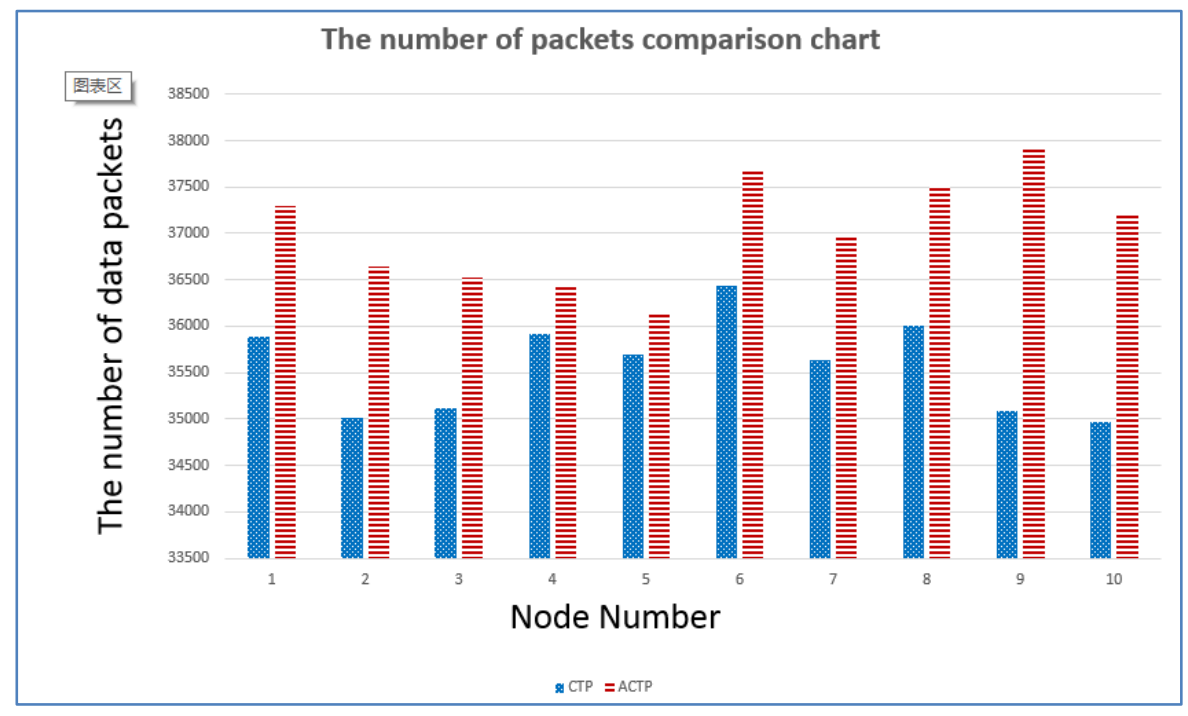

Figure 8. The Number of Packets Comparison Chart 


\section{Conclusions and Future Work}

This paper mainly described the design and implementation of ACTP protocol. CTP only considered the ETX value in selection strategy, but ACTP added two new factors: the residual voltage and the rate of transfer packets. This indeed prolonged the lifetime of the whole networks and the delivery rate of send out packets is also improved. Through the real experiments, we showed that our protocol does achieve the two goals above. In present, the comprehensive probability is determined by the probability of residual voltage and the rate of transfer packets respectively by a factor of 0.5. Later we will consider whether this factor can obtain automatically through the entire networks real environment.

\section{Acknowledgements}

This work is supported by the NSFC (61232016, U1405254, 61173136,61173141, 61173142, 61373133,61502242), 201301030, 2013DFG12860, BC2013012, BY2013095-4-11, the CICAEET fund and the PAPD fund.

\section{References}

[1] I. F. Akyildiz, W. Su, Y. Sankarasubramaniam and E. Cayirci, "A survey on sensor networks", Communications magazine, IEEE, vol. 40, no. 8, (2002), pp. 102-114.

[2] J. Yick, B. Mukherjee and D. Ghosal, "Wireless sensor network survey", Computer networks, vol. 52, no. 12, (2008), pp. 2292-2330.

[3] L. Mo, "Canopy closure estimates with GreenOrbs: sustainable sensing in the forest", Proceedings of the 7th ACM Conference on Embedded Networked Sensor Systems. ACM, (2009).

[4] J. Lundquist, D. Cayan, and M. Dettinger, "Meteorology and hydrology in Yosemite National Park: A sensor network application", Information Processing in Sensor Networks. Springer Berlin Heidelberg, (2003).

[5] R. Fonseca, "The collection tree protocol (CTP)", TinyOS TEP 123 (2006), p. 2.

[6] R. Fonseca, “Tep 123: Collection tree protocol”, Ronald Tutor Hall (RTH), (2006).

[7] R. Fonseca, "Tep 119: Collection”, Core Working Group, TinyOS Community.

[8] Y. Gadallahet, "ECTP: Enhanced Collection Tree Protocol for practical wireless sensor network applications", Wireless Communications and Mobile Computing Conference (IWCMC), 2013 9th International. IEEE, (2013).

[9] Y. Li, H. Chen and R. He, "ICTP: An Improved Data Collection Protocol Based On CTP”, Proceedings of the International Conference on Wireless Communications and Signal, Suzhou, (2010), pp. 1-5.

[10] S. Duquennoy, F. Österlind and A. Dunkels, "Lossy links, low power, high throughput", Proceedings of the 9th ACM Conference on Embedded Networked Sensor Systems. ACM, (2011).

[11] Datasheet T B. Crossbow Inc, (2013).

[12] C. De and S. J. Douglas, "A high-throughput path metric for multi-hop wireless routing", Wireless Networks, vol.11, no. 4, (2005), pp. 419-434

[13] L. Zilong, W. Zou and T. Qi, "A cross-layer congestion control strategy in wireless sensor network", Broadband Network and Multimedia Technology (IC-BNMT), 2011 4th IEEE International Conference on. IEEE, (2011).

[14] J. Zhao, "Load migrating for the hot spots in wireless sensor networks using CTP", Mobile Ad-hoc and Sensor Networks (MSN), 2011 Seventh International Conference on. IEEE, (2011).

[15] J. Zhang, "E-CTP: An Energy-balanced Collection Tree Protocol for Power Constrained Wireless Sensor Networks", International Journal of Grid \& Distributed Computing, vol. 7, no. 2, (2014).

[16] J. Shen, "A Novel Routing Protocol Providing Good Transmission Reliability in Underwater Sensor Networks", Journal of Internet Technology, vol. 16, no. 1, (2015), pp. 171-178.

[17] S. Xie and Y. Wang, "Construction of Tree Network with Limited Delivery Latency in Homogeneous Wireless Sensor Networks", Wireless personal communications, vol. 78, no. 1, (2014), pp. 231-246.

[18] P. Levis, "TinyOS: An operating system for sensor networks." Ambient intelligence. Springer Berlin Heidelberg, (2005), pp. 115-148. 

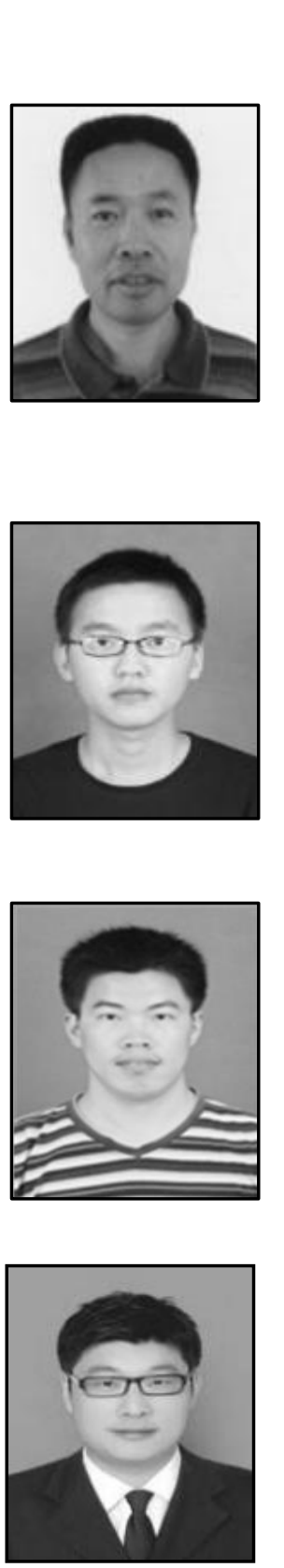

\section{Authors}

Xingming Sun, he is a professor in the School of Computer and Software, Nanjing University of Information Science and Technology, China from 2011. He received the B.S. degree in Mathematical Science from Hunan Normal University and M.S. degree in Mathematical Science from Dalian University of Technology in 1984 and 1988, respectively. Then, he received the $\mathrm{Ph} . \mathrm{D}$. degree in Computer Engineering from Fudan University in 2001. His research interests include information security, network security, cryptography and ubiquitous computing security.

Lei Gao, he received his B.S. degree in Software Engineering from Nanjing University of Information Science and Technology, China in 2012. Currently he is studying for his M.S degree in Software Engineering at the same university. His research interests include wireless networks.

Baowei Wang, he received his B.S. and Ph.D. degrees in Computer Science from Hunan University in 2005 and 2011, respectively. He is currently working as a lecturer in School of Computer and Software, Nanjing University of Information Science and Technology. His research interests include steganography, wireless networks and securing ad hoc networks.

Jian Zhang, he received the B.s degree and M.S. degree in applied mathematics from Qufu Normal University China, in 2005 and Liaoning University, annd 2008, respectively. He received Ph.D degree in Northeastern University, China, in 2012. Currently, he is a Lectrurer in Nanjing University of Information Science \& Technology, China. His research interests include wireless sensor networks, distributed computing, localization and target tracking. 